\title{
La situación hermenéutica del personal docente investigador: Una propuesta para investigar en el campo de la educación desde los prejuicios y la auto-etnografía
}

\author{
The Hermeneutic Situation of the Teacher-Researcher: a proposal to Conduct Research in \\ the Field of Education using Prejudice and Autoethnography
}

Rodrigo Moreno Aponte

Universidad de Málaga, España. rodrigo.moreno81@yahoo.com

Fecha de recepción: 21/4/2015. Reenvios: 8/3/2016 - 23/3/2016.

Fecha de aceptación: 20/5/2016. Fecha de publicación: 13/06/2016.

\begin{abstract}
Resumen: El artículo realiza una aproximación a la metodología hermenéutica en el campo de las Ciencias Sociales. Esta metodología se caracteriza ser una postura que se aleja de la distinción epistemológica clásica entre sujeto y objeto. La experiencia del investigador y su contexto situacional se vinculan con su objeto de estudio. Para el desarrollo de estas ideas se aporta, una descripción de la hermenéutica desarrollada por Hans-Georg Gadamer, enfocada en el concepto de prejuicios. Se realiza el vínculo de estas ideas con la técnica de investigación conocida como auto-etnografía. Finalmente, se hace hincapié en el vínculo de estos dos conceptos con el contexto situacional del personal docente investigador para comprender un fenómeno que tiene que ver directamente con su labor. Por lo tanto, este artículo se enfoca en lo que consideramos puede ser el primer momento de este tipo de indagación, a saber: la reducción hermenéutica por medio de la auto-etnografía por parte del personal docente investigador.
\end{abstract}

Palabras clave: Educación, investigador, Gadamer, prejuicios, auto-etnografía.

\begin{abstract}
The paper uses the hermeneutic methodology in the field of Social Sciences. This methodology is characterized for being a position that departs from the traditional epistemological differentiation between the subject and the object. The experience and situational context of the researcher are linked with the subject of study. To develop these ideas, the author describes hermeneutics as developed by Hans-Georg Gadamer, focused on the concept of prejudice. In the paper, these ideas are related to the research technique known as autoethnography. Finally, emphasis is given to the ties between these two concepts and the situational context of the teacher-researcher in order to understand a phenomenon that is closely related to their work. Therefore, this paper is focused on what we consider may be the first instance of this type of research, namely: hermeneutic reduction through auto-ethnography by the teacher-researcher.
\end{abstract}

Keywords: Education, researcher, Gadamer, prejudice, autoethnography. 


\section{Introducción}

El presente artículo plantea una propuesta hermenéutica para la investigación cualitativa en ciencias sociales, en el campo de la educación, particularmente cuando estos procesos son desarrollados por docentes y su objeto de estudio tiene que ver con la experiencia de estudiantes. Buscaremos sustentar la siguiente afirmación: hacer evidentes los prejuicios del personal docente investigador en educación por medio de su auto-etnografía nos permite acercarnos a la comprensión de un problema en este campo de la investigación en ciencias humanas. Para tal fin, el texto se dividirá en tres momentos. En principio describiremos la idea del prejuicio, concepto central en el pensamiento del hermeneuta Hans-Georg Gadamer, que nos llevará a entender en qué consiste la comprensión de un texto. En segundo lugar, vincularemos las ideas desarrolladas en la primera parte con la descripción de las características de la auto-etnografía como técnica de investigación. Finalmente, mostraremos las conclusiones encaminadas a establecer la relación entre el personal docente investigador en el campo de la educación con los conceptos de las dos primeras partes de este artículo, y las consecuencias metodológicas resultantes de asumir esta postura investigativa.

Uno de los grandes aportes para la comprensión e interpretación de textos en el campo de la hermenéutica se la debemos al filósofo alemán Hans-Georg Gadamer. Su influencia ha permitido desarrollar formas de acceso a la comprensión de diversos problemas, tanto de las humanidades como de las ciencias sociales, teniendo en cuenta que quien lee en busca de comprender un texto no puede partir de una interpretación meramente subjetiva de lo que busca comprender, ni tampoco tiene por qué ceñirse a una interpretación que deviene solamente de la lectura del texto, mucho menos que el acceso al texto parte de la comprensión de la historia de vida del escritor o escritora. La comprensión, más bien, tiene que ver con el vínculo entre el momento histórico de su autoría, el texto y la actualidad del sujeto lector que busca comprender. Esto nos estaría indicando que el acto de comprensión de un texto necesita la implicación de la experiencia factual de quien lee con la historia de vida del autor o autora del texto, y así mismo, con el momento histórico del texto en sí.

Lo anterior, en un plano epistemológico, supone un alejamiento de la clásica división que hacen las ciencias exactas entre sujeto y objeto. Esta idea indica que quien investiga debe distanciarse de su objeto de estudio para hallar una objetividad y permitir que se evidencien los resultados de una forma transparente. De ninguna manera el objeto de estudio debe ser permeado por situaciones externas, entiéndase por esto las ideas preconcebidas del investigador o investigadora, ideologías y contextos históricos. Por tal razón es el mismo objeto el que evidencia los resultados de una investigación.

Aunque Gadamer, con su propuesta hermenéutica, de ninguna manera quiere equipararse al método de las ciencias exactas, tampoco descalifica la importancia del método científico. Más bien busca mostrar que la posición de la comprensión en la hermenéutica va en una vía 
distinta, y llama la atención ante la pretensión de que el metodismo cientifista quiera abarcar todas las formas de expresión de la experiencia. En este sentido, afirma Gadamer (1992): "A medida que los métodos de la ciencia se extienden a todo lo existente resulta más dudoso que los presupuestos de la ciencia permitan plantear la cuestión de la verdad en todo su alcance" (p. 52). Mientras que la hermenéutica busca el acceso a la verdad, las ciencias exactas buscan su acceso a la veracidad, lo demostrable, esto es, la búsqueda de la certeza que les da el método.

Este alejamiento del método también puede llevar al equívoco de suponer que si este no aparece de acuerdo con la forma positiva de las ciencias exactas, entonces se podría caer en un relativismo en el que cualquier comprensión es válida. Para evitar caer en el problema del relativismo, Gadamer propone un estado de alerta para quien quiera comprender un texto. La persona que interpreta debe cuidarse de que sus "ocurrencias" determinen lo que quiere interpretar. No quiere decir esto que debe llegar con su mente en blanco al texto, puesto que es innegable que llega siempre con expectativas de lectura. En consecuencia, aunque nos acerquemos al texto con una idea previa, no puede ser esta la que determina nuestra interpretación. Así pues, estaríamos frente a una situación problemática: El acercamiento hermenéutico a la comprensión de un texto propone un alejamiento de la dicotomía sujeto-objeto propia de las ciencias positivas, puesto que las ideas previas hacen parte del proceso de comprensión del texto, no obstante estos prejuicios pueden desviar la comprensión del texto. A continuación abordaremos esto último para evidenciar que, más que un problema, es una afirmación de la esencia del acto de comprensión.

\section{El control de los prejuicios para la comprensión de un texto}

En la cotidianidad usamos frecuentemente la palabra prejuicio para referirnos a ese momento en el cual tenemos una idea previa de alguna cosa, y que hace que no podamos comprenderla en su esencia puesto que estamos ceñidos a la arbitrariedad de nuestra opinión. De esto deviene que podamos hablar de personas prejuiciosas, esto es, sujetos que tienen arraigadas ideologías que les impiden comprender otras formas de expresión distintas a la de su propia experiencia cultural. Esta situación contiene una compresión subjetiva radical que impide el camino de la interpretación.

Para la hermenéutica de Gadamer, el prejuicio juega un papel vital en el proceso de comprensión de un texto. El prejuicio no posee aquí un carácter negativo, su significado, más bien, es la posición positiva o negativa que tomamos frente a algo. Esto lleva a tener presente que estos prejuicios deben salir a flote cuando buscamos comprender un texto. De esta manera podemos estar al tanto para no caer en una subjetividad radical que oculte el acceso al texto.

Esta situación admite que también debemos dar la voz al texto cuando buscamos comprenderlo. Permitirle "hablar" al texto no supone que hagamos una salida de nuestros prejuicios. La cuestión consiste en que podamos controlarlos y tener presente cuáles nos permiten una mejor comprensión. Por lo tanto, no es que debamos dejar de lado los prejuicios -si fuera de 
esta manera volveríamos a una dicotomía sujeto-objeto-. Lo que se busca desde la propuesta de Gadamer es que para estar al tanto de los prejuicios necesitamos del momento de la precomprensión. Esto implica una comprensión de nosotras o nosotros mismos, de nuestra actualidad y de cómo esta puede mediar en el acto de comprensión. Una vez develados nuestros prejuicios por medio del acto de precomprensión podemos desplazarnos hacia el texto.

Lo que da por sentado el proceso de comprensión es que haya un movimiento hacia el texto a partir del presente de quien lee, pero a la vez, que se permita que el texto evidencie su sentido desde su pasado. "Es claro que la experiencia del tú tiene que ser algo específico por el hecho de que el tú no es un objeto sino que él mismo se comporta respecto a uno" (Gadamer, 2003, p. 434). El texto debe tener voz cuando nos acercamos a él. No tendría sentido el acto de interpretar a partir de nuestros prejuicios incontrolados. Es decir, sabemos que los poseemos y podemos llegar con ellos. Siempre y cuando el prejuicio deje "hablar" al texto, se puede dar la comprensión desde un vínculo entre quien busca comprender y el texto, no desde la radicalización de la idea preconcebida. El texto nos interpela para permitir una comprensión que no clausura su sentido desde su interior o exterior de la interpretación del lector (Roldan, 2012).

El acto de comprensión apunta al horizonte del pasado desde el presente del sujeto lector por medio de una fusión de horizontes: el horizonte del texto y el horizonte de su intérprete. Esto supone que

... cuando nuestra conciencia histórica se desplaza hacia horizontes históricos esto no quiere decir que se traslade a mundos extraños, a los que nada vincula con el nuestro; por el contrario, todos ellos juntos forman ese gran horizonte que se mueve por sí mismo y que rodea una profundidad histórica de nuestra conciencia más allá de las fronteras del presente. En realidad es un único horizonte el que rodea cuanto contiene en sí misma la conciencia histórica. El pasado propio y extraño al que se vuelve la conciencia histórica forma parte del horizonte móvil desde el que se vive la vida humana y que determina a esta como su origen y como su tradición. (Gadamer, 2003, p. 375).

Encontramos acá que estamos ante una situación temporal en una relación pasado y presente. El pasado del texto y el presente de su intérprete. En la comprensión, además del pasado, media la conciencia de que estamos en el mundo; desligarnos de eso sería desvincularnos de nuestro sentido originario como sujetos que tenemos experiencia de la realidad. El texto pertenece no solo a su pasado, sino a una relación con nuestra experiencia de la actualidad.

El control de los prejucios en esta relación temporal no se agota en asumir que tenemos conciencia de estos. Ser conscientes de los prejuicios nos invita a tener presente que hay una distancia temporal en la que tenemos que estar siempre al tanto de las interpretaciones que nos puedan alejar de la comprensión. 
La distancia temporal actúa de foco iluminador del sentido del texto. Ella, en cuanto a situación histórica distinta, resalta aquellos elementos relevantes y significativos para la nueva situación, a la vez que deja en la sombra otros elementos que ya no son expresivos. Se va haciendo así una criba de elementos brutos contenidos materialmente dentro de la tradición, en razón de la nueva situación iluminadora. Así pues, la distancia en el tiempo ejerce la función de filtro sobre los prejuicios erróneos que provocan malentendidos. (Mancilla, 2013, p. 182).

En tal sentido, esta sería una vigilancia constante del lector o lectora frente a su proceso interpretativo a partir de la conciencia que de los prejuicios que alejan del sentido del texto. De esta manera se podría superar la posibilidad de un relativismo que asuma como válidas todas las interpretaciones. El camino hermenéutico que nos propone Gadamer para la comprensión de un texto implica una deliberación acerca de las decisiones que tomamos sobre la comprensión. Es acá donde se juegan los prejuicios y su puesta en evidencia en la fusión de horizontes que permite el vínculo temporal entre quien lee y el texto.

En resumen, los prejuicios vendrían a ser una oportunidad precisa para poder acceder a una comprensión del texto, si se tiene en cuenta que la realidad del lector o lectora y la tradición histórica del texto intervienen en el proceso de comprensión. Entonces, tanto el presente del lector, presente del texto en relación con su pasado y la unión de estos dos por medio de la fusión de horizontes, atacan el problema de una interpretación sesgada por su particular situación cultural, o en un lado opuesto, la interpretación de un texto acudiendo solamente a su contexto en el momento que sale a la luz.

\section{La auto-etnografía como forma de control de los prejuicios}

Hasta aquí hemos expuesto, desde la propuesta de Gadamer, la importancia del prejuicio cuando buscamos comprender e interpretar un texto. Ahora queremos destacar que la hermenéutica, llevada al campo de una investigación cualitativa en el campo de las ciencias humanas, puede hallar en la técnica de la auto-etnografía una herramienta precisa para poner de relieve los prejuicios de quien investiga.

Mientras que la etnografía, a nivel general, realiza descripciones a partir de una observación desde el interior o exterior de un grupo en particular, la auto-etnografía se enmarca dentro de las técnicas de investigación cualitativa que dan cuenta de procesos narrativos del investigador o investigadora en los que acude a la descripción de su propia realidad. La auto-etnografía hace parte de una narración particular por la persona que investiga, para evidenciar cuál es su relación con el contexto cuando participa activamente en este mismo. Este sería el caso de docentes que investigan en un contexto educativo, dada cuenta que están mediados por una serie de creencias, ideologías, teorías u opiniones acerca de un contexto que tiene tantos puntos de análisis. Esto implica una disolución absoluta de la relación sujeto-objeto, para pasar a 
hablar de una relación sujeto-sujeto, sujeto investigador-sujeto investigado, ambos mediados por una misma realidad. Esto permite establecer que estamos ante una relación intersubjetiva entre quien investiga y participantes de esa realidad que se quiere comprender. Por tal razón, la auto-etnografía:

...rompe con los criterios... que focalizan a la investigación etnográfica como un ejercicio en el cual el investigador se centraliza en las evidencias acumuladas por la investigación, se mantiene ajeno alproceso y los acontecimientos que describe, demuestra tener cierta objetividad sobre la realidad que observa, y no refleja ninguna posición personal más allá de la descripción de los hechos y las interpretaciones. La auto-etnografía en cambio sitúa al investigador en el centro de la investigación como un autor integrado, relaciona su reflexión a lo desconocido, y al conocimiento creado por estas relaciones y las evidencias interpretadas y explora lo emotivo y espiritual como acercamientos entre la reflexión personal y las interpretaciones que se suelen hacer a nivel universal. (Montero-Sieburth, 2006, p. 67).

La propuesta de sacar a la luz el contexto situacional del sujeto investigador en relación con la realidad social que busca comprender constituye un desafío para acudir a la propia experiencia para tener una comprensión social más amplia (Scribano y de Sena, 2009). Esto significa reconocer que la realidad del sujeto investigador media de una u otra forma en el proceso investigativo. De no ser porque la idea de lo que se busca investigar llegue por una iluminación extrasensorial, lo cierto es que algo de nuestra experiencia vital nos motiva a buscar un problema que queremos investigar. También situaciones en las que el sujeto investigador se relaciona con la realidad lo llevan a optar por uno u otro horizonte para comprender una situación. La pretensión de la auto-etnografía, entonces:

...no es el acercamiento sensible para "insertarnos" en un contexto determinado, sino la investigación de contextos en los cuales los investigadores también somos actores sociales que vivimos de una manera determinada aquello que es nuestra realidad. Del mismo modo, podemos extender la auto- observación al análisis y exploración critica de los aspectos naturalizados de nuestros contextos específicos de vida. (Sandoval, 2013, p. 42).

Por lo tanto, la auto-etnografía permitiría que la voz de quien investiga se haga presente dentro de la investigación, incluso, para dar cabida a relatos personales o autobiográficos, relatos que conciernen a su proceso personal de descripción del fenómeno que se investiga, claro está, situados en el contexto social y cultural que determine su relación íntima con su objeto de estudio (Blando, 2012). Así, pues, la auto-etnografía la consideramos afín a la compresión tal y como se expuso en la primera pate de este escrito, entendiendo que en el contexto hermenéutico:

...elaborar una anticipación adecuada de la cosa significa elaborar proyectos del entender que sean conformes a la cosa debatida. Esto presupone que la cosa nos concierne; 
que estamos afectados por ella. Aquí no es posible elaborar proyecciones adecuadas a la cosa sin que uno mismo entre en el juego, es decir, sin que uno se ponga dialogar con la cosa. Este modelo dialogal de entender se halla orientado, indudablemente, en contra del paradigma epistemológico de un sujeto del entender que se halle desconectado de su objeto. (Grondin, 2003, p. 139).

Acudir a una descripción personal de ninguna manera puede considerarse como una especie de narcisismo investigativo. Más bien esta propuesta nos puede llevar a comprender que como personas investigadoras no habitamos un Olimpo en el que somos invisibles para el fenómeno particular del mundo de la vida cotidiana que queremos comprender, sino que somos parte activa en ese mundo vital. Debemos reconocer, citando a Gadamer (2003), que "en las ciencias del espíritu el interés del investigador que se vuelve a la tradición está motivado de manera especial por el presente y sus intereses. Sólo en la motivación del planteamiento llegan a constituirse el tema y el objeto de la investigación" (p. 353). Así se sea un científico con un pensamiento positivista extremo, sus intereses parten de su vida como tal, por ende, podríamos entender que de una u otra forma su estado situacional interviene en su proceso investigativo. Si no fuera de esta manera, sencillamente diríamos que es un ser inerte carente de vida que no tiene ninguna forma de experiencia de la existencia. Este tipo de investigador o investigadora, y el sujeto investigador social, se diferencian en que el primer grupo no necesita una precomprensión como sujeto que habita el mundo, ni sacar a la luz sus prejuicios, puesto que estos no intervienen en los resultados de los análisis de su objeto de estudio; el segundo, según hemos expuesto, sí necesita de todo esto para el acto de comprensión.

La propuesta auto-etnográfica se relaciona directamente con la propuesta gadameriana del prejuicio. Mientras que la auto-etnografía nos invita como sujetos investigadores a sacar a la luz nuestra experiencia de mundo que media en la investigación que se quiere llevar a cabo; la hermenéutica llama la atención en la necesidad de comprendernos en nuestra factualidad como sujetos investigadores para poder acceder a la comprensión del texto. La mecánica viene a ser la misma. Por lo tanto, la auto-etnografía guarda la misma esencia de la precomprensión de la hermenéutica, a saber: Desvelar la experiencia del sujeto investigador (prejucios) que media en la lectura de un hecho social permite acceder a la comprensión de un fenómeno social (texto).

\section{De la hermenéutica y la auto-etnografía a la investigación en educación}

Llegados a este punto, vamos a relacionar los conceptos de las secciones precedentes de este texto con un componente investigativo en ciencias humanas como es el campo de la educación. Para esto queremos invitar al lector o lectora a considerar que quien investiga es un ser lector, y su objeto de estudio es un texto. En este sentido queremos asimilar al investigador o investigadora a un sujeto intérprete que está mediado por sus prejuicios, y el objeto de estudio como un texto que dice algo desde su horizonte de comprensión cuando se fusiona con el de 
quien investiga. Queremos apuntar a una situación particular en la investigación en educación, cuando se da el evento en el cual la persona que investiga esta realidad es docente y cuyo objeto de estudio es un fenómeno de la experiencia de sus estudiantes. Por consiguiente, también quisiéramos asimilar la figura del estudiantado a un texto'.

Con esto último queremos decir que como personal docente investigador necesitamos hacer un desplazamiento de nuestra realidad particular hacia la tradición histórica de la experiencia estudiantil para poder acercarnos al acto de comprensión. Como docentes que investigan queremos hacer un acercamiento hermenéutico; en el plano de la educación debemos ir hacia el estudiantado, de manera tal que la interpretación que hagamos de él entre en "conflicto" con nuestros prejuicios. Cada estudiante tiene un cúmulo de experiencias que debemos desentrañar para entender el sentido del fenómeno que queremos comprender. Esto quiere decir, en un sentido hermenéutico, que el estudiantado como texto solo puede ser comprendido cuando nos desplazamos desde nuestra experiencia factual hacia la experiencia que queremos comprender para poder realizar la fusión de horizontes.

Resulta claro que como docente investigador estoy mediado por un contexto que comparto con el estudiantado. Esto no quiere decir que nuestra experiencia sea la misma. Tenemos puntos de vista que en algunos casos llegan a ser antagónicos, a pesar de referirnos a la misma cosa, a tal punto de parecer vivir en un espacio-tiempo distinto, casi como personas desconocidas. ¿Qué genera el desconocimiento en esta relación personal docente investigador y estudiante que se convierte en dicotómica?

Los métodos de investigación en educación que asumen el conocimiento total de la experiencia estudiantil desconocen la situación hermenéutica -no tienen que conocerla en realidad, dada su teleología-. Alejarnos de ese plano nos lleva a que la experiencia de la verdad desde el plano hermenéutico haga que salgamos, como investigadores o investigadoras, de nuestro ego para desplazarnos a la situación histórica del estudiantado. Por esta razón estaríamos guardando un sentido afín con la siguiente idea:

Desde la experiencia hermenéutica concebimos la educación, por tanto, como una tarea esencial de mediación a la que la coyuntura epistemológica de la racionalidad ha destituido de sus funciones principales. Sólo han interesado las mediaciones más instrumentales como la socializadora, por una parte, que convierte a la educación en mera actividad de reproducción de normas, costumbres, valores y prácticas, como la estrictamente científica, por otra, que permite y posibilita la transmisión de esas destrezas simbólicas y técnicas del grupo en su instalación impositiva y dominadora sobre el entorno de sí mismo. (Esteban, 2002, p. 107).

$1 \quad$ La noción de texto en la hermenéutica fue trasladada al estudio de otros aspectos de la realidad. Esto nos permite hablar de texto no solo refiriéndonos a un escrito. "La universalidad del problema hermenéutico, reconocida ya por Schleiermacher, abarca todo lo racional, todo aquello que puede ser objeto de acuerdo mutuo."(Gadamer, 1992, p.392). 
De una u otra forma, con lo expuesto hasta ahora, se ha buscado evidenciar que una de las necesidades hermenéuticas de quienes investigan en el campo de las ciencias humanas es trascender nuestro propio plano cultural, sin dejarlo de lado, por supuesto, para insertarnos en una experiencia inmediata del estudiantado. "El simple hecho de preguntarse por la posibilidad de trascender los propios parámetros culturales, manifiesta, de algún modo, que ya se ha hecho. Pues para formular esta pregunta hay que situarse en un marco de referencia más amplio, y solo desde ahí se puede plantear la cuestión acerca de la naturaleza de las representaciones y las mediaciones en cuanto tales" (García, 2002, pp. 125-126). Trascender el plano cultural no es negarlo, sería absurdo hacer semejante aseveración, por lo contrario, querer ir más allá de este invita a tenerlo en cuenta.

Como lo hemos visto, una situación hermenéutica no deja de lado el contexto; pero no se puede negar que la situación hermenéutica invita a ir más allá de este. Esto quiere decir que un análisis hermenéutico en educación podría partir de la descripción del contexto situacional para dar paso al conocimiento directo de la experiencia vivida.

Sin duda, nuestra concepción del mundo depende de las ideas que hemos heredado de nuestra tradición cultural, y diferentes sociedades y grupos humanos ven la realidad de formas muy di versas en aspectos importantes. Pero ésta no es razón suficiente para negar que haya algunas características del mundo cuya existencia es independiente de nosotros que permiten que podamos establecer distinciones entre unos modos de percibir y otros. (García, 2002, p. 128).

Cuando se está en la situación de investigar en el campo de la educación, cumpliendo a la vez el rol de docentes del espacio de experiencia que queremos comprender, deberíamos tener en cuenta de antemano que esto nos permite interactuar con el objeto de estudio, y de esta manera podemos formularnos preguntas y conocer los problemas desde otros ángulos. Por lo tanto, se debe tener en cuenta que de ninguna manera el individuo investigador es invocado por un fenómeno social debido a sus grandes conocimientos superiores, sino porque hace parte de una comunidad, grupo de personas o situación susceptible de ser observada (Scribano y de Sena, 2009). Por consiguiente, tener en cuenta los prejuicios por medio de una auto-etnografía como docentes que investigan en el campo de la educación nos permite situarnos ante ellos, controlarlos, y tenerlos presentes para desplazarnos a la experiencia del estudiantado.

En síntesis, en el recorrido que hicimos a lo largo de este texto buscamos evidenciar la importancia de que en una investigación que tenga como presupuestos metodológicos a la hermenéutica, y como base los prejuicios, se halle la voz de quien investiga desde su experiencia en relación con el fenómeno social que investiga. Las consecuencias de asumir una investigación de esta manera, implican tener presente como investigadores una postura que va en clara oposición a los paradigmas positivistas que asumen una distancia entre el sujeto investigador y su objeto de estudio (sujeto-objeto). 
Nuestro objeto de estudio se tiene en cuenta no como la cosa que puedo manipular, sino como un complejo cúmulo de experiencias que requieren que nos instalemos dentro de situaciones de mundo, algunas veces disímiles, otras afines a la nuestra. Por lo tanto, no cabría acá una postura en la que nos asumamos como en un estadio del conocimiento más arriba de quien comparte sus experiencias para llevar a cabo nuestra indagación. Consideramos que mover los hilos de otra persona para nuestros fines investigativos sería cosificar, fragmentar y, de por sí, aniquilar la experiencia del fenómeno que se busca comprender.

Acudir a una investigación en ciencias sociales en el campo de la educación, en calidad de docentes investigadores o investigadoras que quieren comprender la experiencia de sus estudiantes, supone situarnos en la idea de que somos sujetos y objetos a la vez. No nos podemos desentender de uno u otro. Hacerlo sería asumir que estamos en una jerarquía con respecto al entorno al cual queremos aproximarnos.

\section{Referencias}

Esteban, J. (2002). Memoria, hermenéutica y educación. Madrid: Editorial Biblioteca Nueva.

Gadamer, H. G. (1992). Verdad y método II. Salamanca: Editorial Sígueme.

Gadamer, H. G. (1995). La idea de la filosofía práctica. En El giro hermenéutico. Madrid: Editorial Cátedra.

Gadamer, H. G. (1995b). Ciudadano de dos mundos. En El giro hermenéutico. Madrid: Editorial Cátedra.

Gadamer, H. G. (1995c). Razón y filosofía práctica. En: El giro hermenéutico. Madrid: Editorial Cátedra.

Gadamer, H. G. (2003). Verdad y método I. Salamanca: Editorial Sígueme.

García, M. (2002). La educación, actividad interpretativa. Hermenéutica y filosofía de la educación. Madrid: Editorial Dikinson.

Grondin, J. (2003). Introducción a Gadamer. Barcelona: Editorial Herder.

Mancilla, M. (2013). Experiencia de la historicidad e historicidad de la experiencia: El mundo como espacio hermenéutico. Revista Alpha (36). doi: 10.4067/S0718-22012013000100012.

Montero-Sieburth, M. (2006). La auto etnografía como una estrategia para la transformación de la homogeneidad a favor de la diversidad en la escuela. Memorias del Congreso Internacional de Educación Intercultural. Formación del profesorado y práctica escolar. Recuperado de http://www.uned.es/congreso-inter-educacion-intercultural/Grupo discusion 1/74.pdf 
Sandoval, J. (2013). Una perspectiva situada de la investigación cualitativa en ciencias sociales. Revista Cinta de Moebio, (46), 37-46. Recuperado de http://www.moebio.uchile.cl/46/ sandoval.html

Scribano, A. y de Sena, A. (2009). Construcción de conocimiento en Latinoamérica: Algunas reflexiones desde la auto-etnografía como estrategia de investigación. Revista Cinta de Moebio, (34), 1-15. Recuperado de http://www.moebio.uchile.cl/34/scribano.html 